\title{
Healthcare-Related Costs Associated with Switching Subcutaneous Tumor Necrosis Factor- $\alpha$ Inhibitor in the Treatment of Inflammatory Arthritis: a Retrospective Study
}

\author{
Johan Dalén · Karin Luttropp · Axel Svedbom • Christopher M. Black • \\ Sumesh Kachroo id
}

Received: June 1, 2020 / Published online: July 9, 2020

(C) The Author(s) 2020

\begin{abstract}
Introduction: Subsequent lines of subcutaneous tumor necrosis factor alpha inhibitor (SCTNFi) treatment may be well motivated in the management of rheumatoid arthritis (RA), ankylosing spondylitis (AS), and psoriatic arthritis (PsA) - collectively named inflammatory arthritis (IA). However, the costs associated with switching SC-TNFis are largely unknown. The objective of this retrospective observational study was to explore costs of healthcare resource utilization (HCRU) associated with switching SC-TNFi treatment among biologicnaïve Swedish patients with IA.

Methods: Using population-based register data, adult patients filling prescriptions between May 6, 2010 and December 31, 2014 for an SCTNFi (adalimumab, etanercept, certolizumab, and golimumab) were included. Patients switching treatment (cyclers) were matched to treatment persistent patients on the basis of propensity score and follow-up time. HCRU-
\end{abstract}

Digital Features To view digital features for this article go to https://doi.org/10.6084/m9.figshare.12563852.

J. Dalén · K. Luttropp · A. Svedbom

ICON plc, Stockholm, Sweden

C. M. Black · S. Kachroo ( $\square)$

Center for Observational and Real-World Evidence,

Merck \& Co., Inc, Kenilworth, NJ, USA

e-mail: sumesh.kachroo@merck.com associated costs were captured and compared 12 months before and 12 months after the index date (defined as the date of the switch).

Results: A balanced cohort of 594 matched pairs was derived. Prior to the index date, cyclers had significantly higher non-treatment HCRU costs compared to persistent patients (\$3815 [3498-4147] vs. \$2900; 95\%CI [2565-3256]). However, 12 months after the index date, cyclers had significantly increased their non-treatment HCRU costs while persistent patients lowered theirs (\$822 [232-1490] vs. $\$-313[-664-36])$. This resulted in a statistically significant difference in difference of $\$ 1135$ between the groups.

Conclusions: In biologic-naïve patients treated with SC-TNFi for IA, cyclers significantly increased their non-treatment HCRU costs 12 months after switching treatment while persistent patients lowered their costs during the same time period. As these findings indicate that differences in treatment persistence may have an impact on costs, further research utilizing more comprehensive data sources in alternate settings is warranted.

Keywords: Ankylosing spondylitis; Biologics; Cost; Inflammatory arthritis; Psoriatic arthritis; Rheumatoid arthritis; Rheumatology; Subcutaneous TNF $\alpha$ inhibitors; Switching; Treatment persistence 


\section{Key Summary Points}

Few studies have compared healthcare resource utilization and costs in patients with inflammatory arthritis (IA) who switch from a first-line to a second-line SC-TNFi to those who remain on their first-line subcutaneous tumor necrosis factor alpha inhibitor (SC-TNFi) treatment.

The body of literature is concentrated to the USA and the costs associated with switching biologics in a European setting is unknown.

To our knowledge, no study has measured costs before and after the occurrence of a treatment switch among patients matched for baseline characteristics and duration of first-line treatment, thereby accounting for differences between the groups that may otherwise bias estimates of costs attributable to switching.

The results show that in biologic-naïve patients treated with SC-TNFis for IA, patients switching treatment significantly increased their non-treatment healthcare resource utilization (HCRU) costs 12 months after switching treatment; meanwhile, persistent patients significantly lowered their costs.

The results of this study may further guide physicians and payers in optimizing treatment decisions from clinical and economic perspectives.

\section{INTRODUCTION}

Ankylosing spondylitis (AS), psoriatic arthritis (PsA), and rheumatoid arthritis (RA) - collectively inflammatory arthritis (IA)-are rheumatic conditions characterized by chronic inflammation, due in part to an elevated level of tumor necrosis factor- $\alpha$ (TNF $\alpha$ ). These autoimmune disorders affect joints and organs, resulting in pain, deformity, and disability [1-2], thereby conferring a great burden from both a patient and health economic point of view [3-4]. The IA treatment landscape has evolved substantially over the past two decades [5], as biologic treatments such as TNF $\alpha$ inhibitors (TNFi) have been added to the existing conventional disease-modifying anti-rheumatic drugs (DMARDs) and non-steroidal anti-inflammatory drugs (NSAIDs).

In the event of a treatment failure due to loss of response and/or one or several adverse events deemed unacceptable, patients who have received first-line treatment with a subcutaneous TNFi (SC-TNFi) may switch to secondline treatment with another type of SC-TNFi $[6,7]$. While a switch from a first-line to a second-line SC-TNFi treatment may be well motivated from a clinical viewpoint, the costs associated with switching treatments are largely unknown. Few studies have compared healthcare resource utilization (HCRU) and costs in patients with IA who switch from a first-line to a second-line SC-TNFi ("cyclers") to those who remain on their first-line SC-TNFi treatment [8-10], especially in European populations. Furthermore, to the best of our knowledge, no study has measured costs before and after the occurrence of a treatment switch among a set of patients matched for duration of first-line treatment as well as for baseline characteristics, thus accounting for differences between cyclers and treatment persistent patients which may otherwise bias estimates of costs attributable to switching. More accurate information on costs attributable to switching per se may guide physicians and payers in optimizing treatment decisions from clinical and economic perspectives. Therefore, utilizing the Swedish Health Data Registers, this study aimed to estimate the costs associated with switching among biologicnaïve patients treated with SC-TNFi for IA.

\section{METHODS}

Available upon ethics approval, Swedish administrative data have been used extensively in population-based research. The National 
Health Data Registers (NHDRs), held and maintained by the National Board of Health and Welfare (NBHW), are particularly well suited to investigate research questions pertaining to healthcare owing to their high level of completeness and quality overall $[11,12]$. Swedish healthcare is publicly available and tax funded, with a low level of patient co-payments $[13,14]$. Moreover, Swedish patients with IA receive their systemic treatment prescriptions from specialized rheumatologists, who do not encounter any restrictions in terms of prescribing approved drugs. Hence, there are very few economic incentives weighing in on the choice of treatment, thereby minimizing bias associated with financial considerations on switching treatment.

\section{Data Sources}

Three administrative registers were utilized in this study. The National Inpatient Register (NPR) and the Prescribed Drug Register (PDR), both NHDRs, were complemented with data from the Cause of Death Register (CDR). The last of these records all deaths that occur in Sweden, as well as their underlying cause. The NPR contains data on diagnosis, inpatient stays and their duration, and specialized outpatient care visits, while the PDR contains data on all filled prescriptions dispensed in Swedish pharmacies since June 1, 2005. However, the PDR does not contain any information on medication dispensed in a hospital setting. The NPR was used to extract information on HCRU in conjunction with the PDR, which was used to obtain information regarding SC-TNFi treatment. Meanwhile, the CDR was used to monitor vital status of the included patients throughout the study period. Information from each register was linked by the NBHW through the use of personal identification numbers, which are specific to each Swedish citizen. The authors of this study did not have access to the personal identification numbers and did not participate in linking information from the different registers. As a result of the retrospective non-interventional study design and the applicable laws and regulations pertaining to the utilized data sources, informed consent and participant consent were not applicable. The study was performed in accordance with the Helsinki Declaration and, prior to initiation, ethics approval was granted by the Regional Ethical Review Board in Stockholm, Sweden (approval number 2016/1576-32).

\section{Patient Selection}

An overview of the study design is depicted in Fig. 1. The inclusion and exclusion criteria were applied to identify TNFi biologic-naïve adult patients initiating treatment with SC-TNFi for IA. To be an eligible candidate, patients had to initiate treatment with any of the following SCTNFi between May 6, 2010 (when all currently available SC-TNFis were included in the Swedish reimbursement system) and December 31, 2014: adalimumab, etanercept, certolizumab pegol, or golimumab.

The following exclusion criteria were applied: (1) age less than 18 years at treatment initiation; (2) administration of a TNFi in an inor outpatient hospital setting; (3) a filled SCTNFi prescription from a department other than rheumatology, orthopedics, or rehabilitation; or a prescription issued by a non-rheumatologist; and/or (4) no record of an IA diagnosis in accordance with an algorithm previously presented in Dalén et al. 2016. and Dalén et al. 2017 [15, 16]. The second exclusion criterion was applied to minimize the risk of erroneous estimates of treatment duration due to misleading data gaps in the PDR pertaining to patients receiving TNFi treatment in an in- or outpatient hospital setting. However, for SCTNFi treatments those gaps should be minimal since $99 \%$ of SC-TNFi prescriptions are dispensed from pharmacies [17]. The same criterion also served to exclude patients treated with biologics administered intravenously in a hospital setting.

\section{Stratification and Matching}

To estimate the costs associated with switching, eligible patients were divided into two groups based on first-line SC-TNFi treatment 


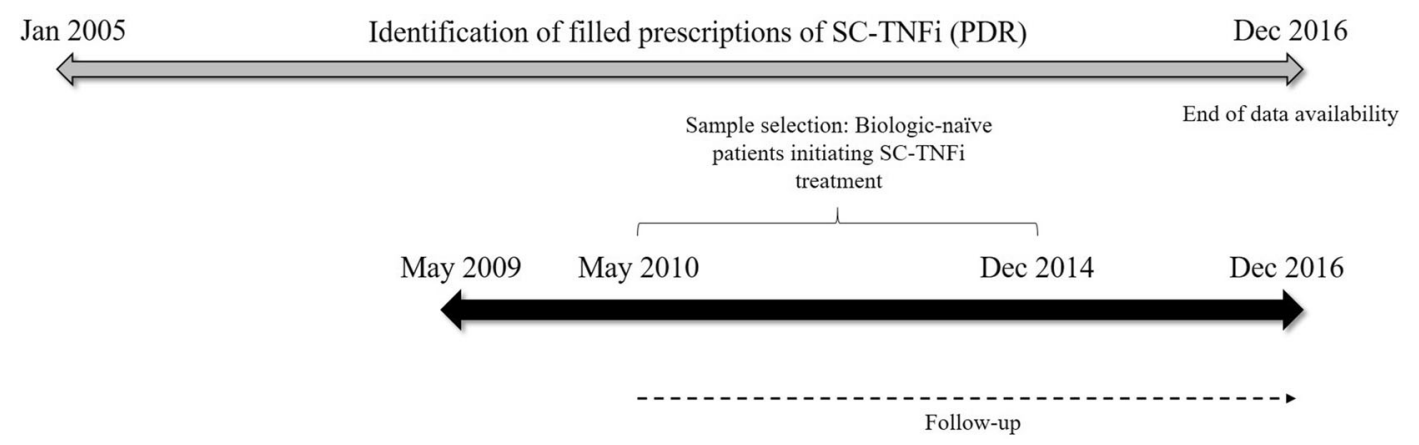

Fig. 1 Study design

persistence status: persistent patients and cyclers. Persistence status was defined in accordance with previous studies $[15,16,18]$, which in turn based their definition of persistence on the work of the ISPOR Medication Compliance and Persistence Work Group [19]. Persistence was derived as the time elapsed between treatment initiation and treatment discontinuation, while allowing for treatment gaps of 60 days between prescriptions. The duration of each prescription was derived by multiplying the number of packages dispensed by the defined daily dose (DDD). A switch to second-line treatment was defined as a filled prescription for an SC-TNFi other than the first-line SC-TNFi within 60 days after discontinuing first-line treatment. Patients were allowed to have a 60-day gap between the end date of the days' supply for the first treatment and the dispensing date for the second treatment before they were considered to be non-persistent. In accordance with the applied definition, patients that at some point during the study period switched to a second-line SC-TNFi treatment were defined as "cyclers". In order to exclude patients not responding during the induction period, only those cyclers reaching the maintenance period (defined to occur after 6 months continuous first-line treatment) before switching were considered. The remaining patients, who did not switch to a second-line SC-TNFi, were eligible to be included as persistent patients with their actual inclusion depending on their persistence status at the relevant point in time.

To account for potential selection bias, the analysis was conducted on propensity score matched (PSM) cohorts, a statistical method often used in observational studies to match cases and controls on a number of covariates using a single scalar-the propensity score $[20,21]$. In sequential steps based on first-line persistence, cyclers (cases) were matched, without replacement, on propensity score to persistent patients (controls). In order to facilitate comparisons across the same time period after first-line treatment initiation, cyclers were grouped by first-line treatment persistence (6-12 months, $12-18$ months, $18-24$ months, etc.) and subsequently matched to controls who were persistent with first-line treatment by at least an additional 12 months. Thus, cyclers persistent for 6-12 months before switching were matched to controls persistent with firstline treatment for at least 24 months; cyclers persistent for 12-18 months before switching were then matched to the remaining sample set of controls persistent with first-line treatment for at least 30 months, etc. This process was repeated until no more matches could be successfully made. The index date was derived from the number of days from treatment initiation to switch for cyclers and assigned to both cases and controls. Hence, persistence with first-line treatment until index date was the same between cases and controls and, consequently, the follow-up period in relation to treatment initiation was exactly the same.

\section{Study Outcomes}

Costs associated with switching SC-TNFi were operationalized and studied as the cost of HCRU captured continuously by month, as well as 
accumulated costs during the 12-month periods before and after the date of switch (defined as the index date). Available healthcare resources comprised of specialized outpatient care, inpatient stays, and non-DMARD medication. The utilization and cost of traditional and biologic DMARDs (henceforth collectively referred to as DMARDs) was also available but was studied separately. The development of continuous costs, measured during 24 months around the index date, was analyzed descriptively only, while the accumulated costs were compared and tested for differences within as well as across cyclers and persistent patients. All costs were converted to and presented in year 2016 US dollars (USD; \$). The included patients were further described using the following baseline characteristics, captured during a period of 12 months prior to SC-TNFi treatment initiation: age, gender, year of treatment initiation, IA type, Charlson comorbidity index (CCI), NSAID use, traditional DMARD use, steroid use, and hospitalizations. All data management and statistical analyses in this study were conducted using SAS ${ }^{\circledR} 9.4$ software (Copyright $\odot 2016$ by SAS Institute Inc., Cary, NC, USA).

\section{Statistical Analysis}

All study outcomes were analyzed descriptively. Frequencies and percentages were provided for dichotomous variables, while continuous variables were described using mean and standard deviation (SD). The results were presented for all included patients and were stratified by persistence with first-line treatment, as described in the "Patient Selection" and "Stratification and Matching" sections. Baseline characteristics were compared across strata, and appropriate tests (e.g., Wilcoxon-Mann-Whitney test, Kruskal-Wallis test, $\chi^{2}$ test, Wilcoxon signedrank test and $t$ test) were implemented on the basis of the number of strata and the distributions of the variables. As a result of the skewed nature of costs associated with HCRU, this outcome was compared using bootstrapped 95\% confidence intervals (CIs) based on unrestricted random sampling with replacement and confidence limits derived from percentiles. The propensity score used to obtain the PSM cohorts was derived from a logit model as the predicted probability of switching to second-line SC-TNFi treatment given the baseline characteristics presented in Table 1. Pairs were matched using a Greedy algorithm (i.e., when a match was made it was not reconsidered) using 5 to 1 digit matching. As suggested by Ho, Imai et al., and Austin [22-24], equality or balance across strata was evaluated by standardized differences in covariates pre- and post-matching rather than $p$ values. Rosenbaum and Rubin's [25] suggested definition of balance was applied, i.e., standardized differences below 0.1 were accepted. Persistent patients and cyclers were matched for age, gender, type of first-line SC-TNFi treatment, first-line treatment duration prior to switch date, and hospitalization (yes/no).

\section{Sensitivity Analysis}

In addition to the main analysis, a separate sensitivity analysis was performed. In the main analysis, persistent patients were required to remain persistent with their first-line treatment throughout the full 24-month follow-up period, while cyclers were allowed to discontinue their second-line SC-TNFi during the 12 months following the index date. The rationale for this difference between patient groups was to get a more complete assessment of the HCRU costs associated with the actual switching of SC-TNFi treatment, as opposed to capturing costs incurred by cyclers who remain persistent with their second-line treatment throughout the followup period only. In the sensitivity analysis, however, cyclers were required to remain persistent with their second-line treatment throughout the 12 months following the index date.

\section{RESULTS}

\section{Study Population}

As depicted in Fig. 2, a total of 15,700 biologicnaïve patients initiated treatment with an SCTNFi for IA between May 6, 2010 and 
Table 1 Baseline characteristics of propensity score matched cohort

\begin{tabular}{lccc}
\hline & $\begin{array}{l}\text { Persistent patients } \\
n=594\end{array}$ & $\begin{array}{l}\text { Cyclers } \\
n=594\end{array}$ & $p$ value \\
\hline Age, mean (SD) & $50.53(14.07)$ & $49.58(13.92)$ & 0.249 \\
Female, $n$ (\%) & $400(67.3)$ & $400(67.3)$ & 1.000 \\
Treatment initiation year, $(n \%)$ & & & $0.057^{\dagger}$ \\
2010 & $97(16.3)$ & $87(14.6)$ & \\
2011 & $145(24.4)$ & $137(23.1)$ & \\
2012 & $131(22.1)$ & $104(17.5)$ & \\
2013 & $141(23.7)$ & $157(26.4)$ & \\
2014 & $80(13.5)$ & $109(18.4)$ & \\
Diagnosis, $n$ (\%) & $153(25.8)$ & $153(25.8)$ & \\
Psoriatic arthritis & $127(21.4)$ & $141(23.7)$ & \\
Ankylosing spondylitis & $314(52.9)$ & $300(50.5)$ & 0.162 \\
Rheumatoid arthritis & $0.83(1.07)$ & $0.80(1.20)$ & \\
CCI, mean (SD) & & $451(75.9)$ & 0.207 \\
Co-medication, $n$ (\%) & $432(72.7)$ & $488(82.2)$ & 0.395 \\
NSAIDs & $499(84.0)$ & $370(62.3)$ & 0.097 \\
DMARDs & $342(57.6)$ & $92(15.5)$ & \\
Steroids & $93(15.7)$ & & \\
Hospitalized, $n$ (\%) & & & \\
\hline
\end{tabular}

$C C I$ Charlson comorbidity index, DMARDs disease-modifying anti-rheumatic drugs, NSAIDs non-steroidal anti-inflammatory drugs, $S C-T N F i$ subcutaneous tumor necrosis factor- $\alpha$ inhibitor, $S D$ standard deviation $\dagger \chi^{2}$ test

December 31, 2014. After applying the exclusion criteria, 8031 patients remained eligible; 1371 were identified as cyclers while 6660 were identified as eligble persistent patients. Out of the cyclers, 766 (56\%) reached the maintainance period prior to switching SC-TNFi. A balanced PSM cohort, with 594 matched pairs, was generated as cyclers vs. persistent patients. Baseline characteristics for the PSM cohort are shown in Table 1 . The mean age across cyclers and persistent patients was 50 years and the majority of the included patients were women (67\%). The distribution of IA diagnosis was also similar, with approximately half of the patients being diagnosed with RA, while PsA and AS each accounted for nearly a quarter of the patients. The average CCI was around 0.8 across the cohort, and a little more than $15 \%$ of patients had been hospitalized during the baseline period. In addition, the use of NSAIDs and use of traditional DMARDs were distributed evenly across the cohort during the baseline period (approximately $75 \%$ and $80 \%$, respectively). Slight numerical differences were observed pertaining to year of treatment initiation, as well as dispensed prescriptions of steroids. However, the standardized differences were within the applied acceptance threshold. 


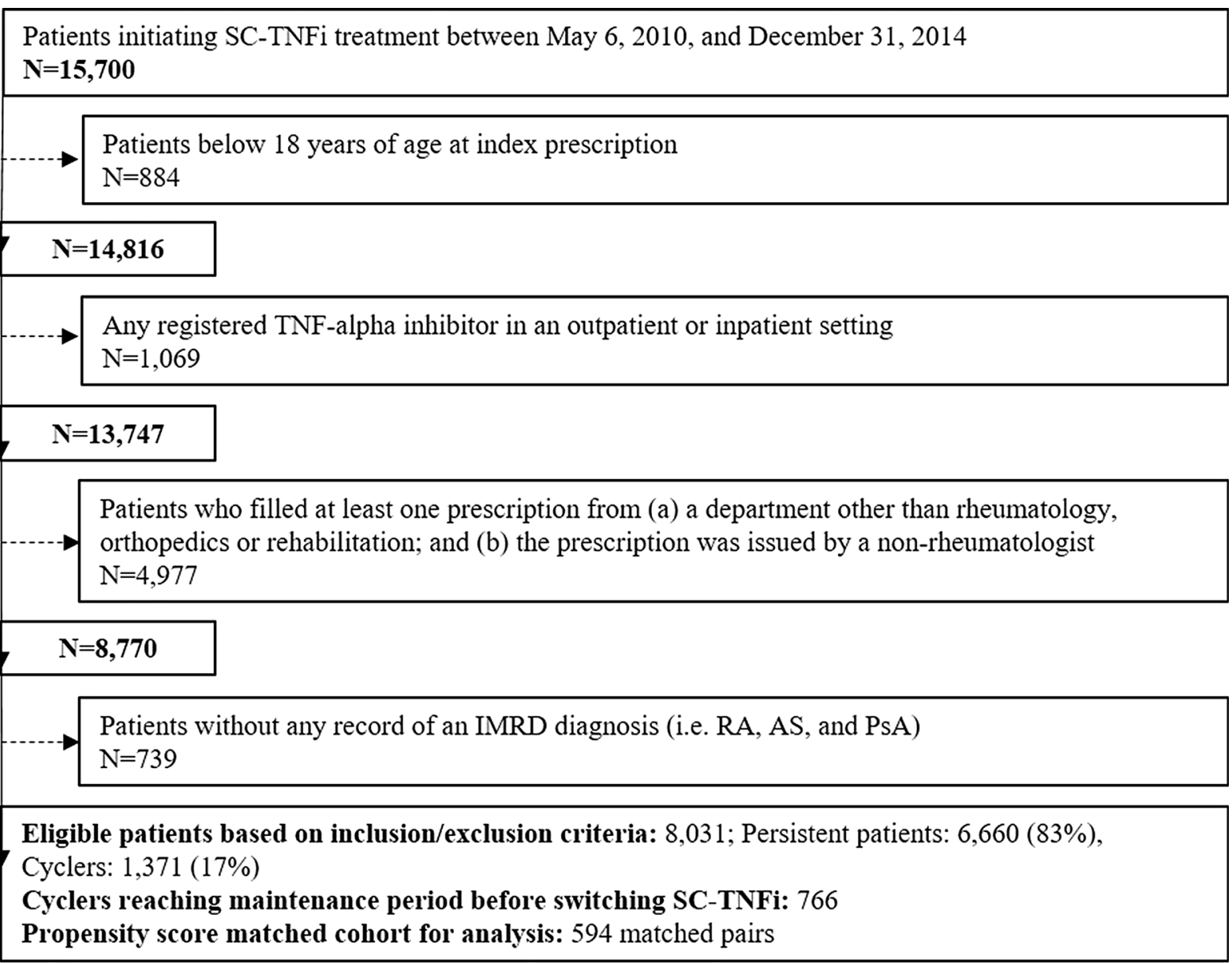

Fig. 2 Patient selection flow chart. IMRD immune-mediated rheumatic disease

\section{Non-Treatment-Related Healthcare Resource Utilization}

Figure 3a-d illustrates the development of HCRU costs excluding DMARD treatment during the 24-month measurement period surrounding the index date, for cyclers and persistent patients, respectively.

The mean cost of specialized outpatient care was similar between cyclers and persistent patients up until a few months before index date (Fig. 3a). Thereafter, persistent patients decreased their mean cost for specialized outpatient care, stabilizing at around $\$ 100$ per month, while cyclers remained stable at a monthly mean cost of around \$200 throughout the follow-up period. In addition, cyclers had a notable $50 \%$ increase in specialized outpatient care costs right before switching. A similar pattern was repeated for costs pertaining to inpatient care (Fig. 3b). Whilst costs were similar between cyclers and persistent patients prior to the index date-slightly below $\$ 100$ per month-cyclers had higher costs for inpatient care during the majority of the follow-up period. Costs for non-DMARD medication were similar across cyclers and persistent patients throughout the follow-up period, at a level of approximately $\$ 50$ per patient and month (Fig. 3c). The development of mean total costs due to non-treatment-related HCRU was largely driven by costs for specialized outpatient and inpatient care and, hence, followed a similar pattern (Fig. 3d).

The mean total costs for non-treatment HCRU accumulated over the 12 months before 

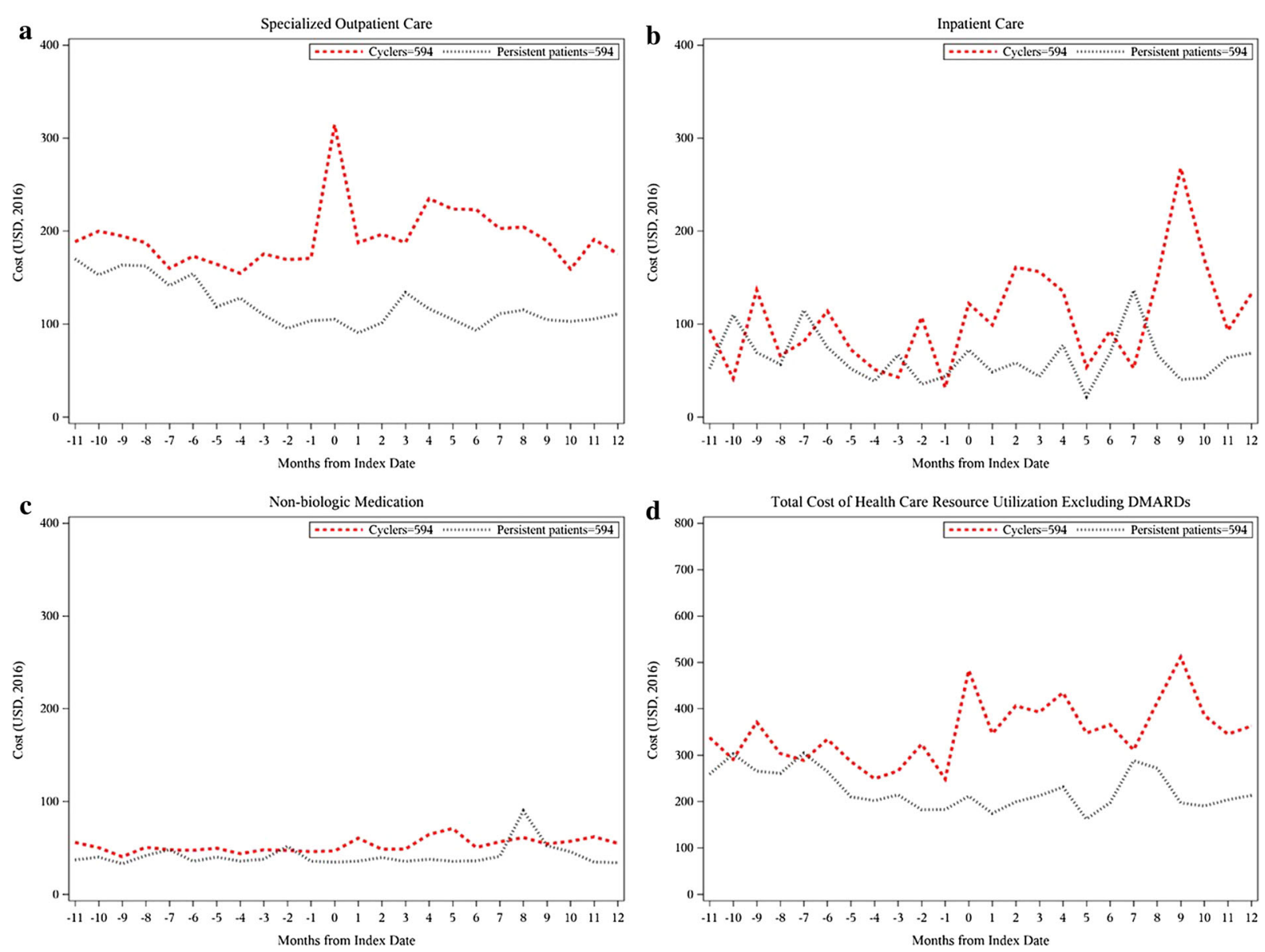

Fig. 3 Non-treatment-related HCRU costs of propensity score-matched persistent patients and cyclers. a Specialized outpatient care. b Inpatient care. c Non-DMARD medication. d Total non-treatment-related HCRU

and 12 months after the index date are presented by persistent patients and cyclers in Table 2. While the costs of inpatient care and non-DMARD medication were similar during the 12 months prior to index date, specialized outpatient care costs were significantly lower among persistent patients than among cyclers. The difference in costs for specialized outpatient care also drove the significant difference in total cost excluding DMARDs prior to index date (\$2900; 95\% CI [2565-3256] vs. \$3815 [3498-4147]).

While treatment persistent patients decreased their mean total non-treatment HCRU costs between the 12 months before and the 12 months after the index date, cyclers increased their costs (\$2587 [2250-2968] vs. $\$ 4637$ [4078-5262]). The mean costs of specialized outpatient care and inpatient care were significantly lower among persistent patients than among cyclers $(\$ 1307$ [1190-1433] vs. \$2414 [2230-2597], and \$750 [533-993] vs. \$1524 [1056, 2102], respectively). Although persistent patients significantly decreased their costs for specialized outpatient care $(\$-327$ [ -432 to -222$])$, this was not enough to generate a significant decrease in total non-treatment HCRU costs $(\$-313$ [ -664 to 36]). Meanwhile, cyclers significantly increased their costs for inpatient care and nonDMARD medication (\$583 [57-1137], and \$118 [40-212], respectively), resulting in a significant increase in total non-DMARD costs (\$822 [232-1490]). The within-group differences yielded a significant difference in difference (DID) of $\$ 1135$ between cyclers and persistent 
Table 2 Healthcare resource utilization costs in propensity score-matched persistent patients and cyclers

\begin{tabular}{|c|c|c|c|c|c|c|}
\hline & \multicolumn{3}{|c|}{$\begin{array}{l}\text { Persistent patients } \\
n=594\end{array}$} & \multicolumn{3}{|l|}{$\begin{array}{l}\text { Cyclers } \\
n=594\end{array}$} \\
\hline & Mean USD & (SD) & {$[95 \% \mathrm{CI}]$} & Mean USD & (SD) & {$[95 \% \mathrm{CI}]$} \\
\hline \multicolumn{7}{|l|}{ HCRU costs 1 year pre-index } \\
\hline Specialized outpatient care & 1634 & $(1460)$ & {$[1515,1762]$} & 2293 & $(1858)$ & {$[2148,2450]$} \\
\hline Inpatient care & 787 & $(3152)$ & {$[540,1054]$} & 941 & $(2904)$ & {$[732,1199]$} \\
\hline Non-DMARDs & 479 & $(863)$ & {$[417,555]$} & 581 & (691) & {$[527,642]$} \\
\hline Total excl DMARDs & 2900 & $(4325)$ & {$[2565,3256]$} & 3815 & $(3924)$ & {$[3498,4147]$} \\
\hline \multicolumn{7}{|l|}{ HCRU costs 1 year post-index } \\
\hline Specialized outpatient care & 1307 & $(1513)$ & {$[1190,1433]$} & 2414 & $(2318)$ & {$[2230,2597]$} \\
\hline Inpatient care & 750 & $(2930)$ & {$[533,993]$} & 1524 & $(6338)$ & {$[1056,2102]$} \\
\hline Non-DMARDs & 530 & $(1636)$ & {$[423,679]$} & 698 & $(1279)$ & {$[602,811]$} \\
\hline Total excl DMARDs & 2587 & $(4403)$ & {$[2250,2968]$} & 4637 & (7494) & {$[4078,5262]$} \\
\hline \multicolumn{7}{|c|}{ Difference in HCRU costs pre- and post-index } \\
\hline Specialized outpatient care & -327 & $(1342)$ & {$[-432,-222]$} & 121 & $(2406)$ & {$[-66,312]$} \\
\hline Inpatient care & -37 & $(3708)$ & {$[-350,233]$} & 583 & $(6656)$ & {$[57,1137]$} \\
\hline Non-DMARDs & 51 & $(1508)$ & {$[44,201]$} & 118 & $(1106)$ & {$[40,212]$} \\
\hline Total excl DMARDs & -313 & $(4487)$ & {$[-664,36]$} & 822 & $(7588)$ & {$[232,1490]$} \\
\hline
\end{tabular}

$C I$ confidence interval, $H C R U$ healthcare resource use, DMARDs disease, modifying anti-rheumatic drugs, SC-TNFi subcutaneous tumor necrosis factor- $\alpha$ inhibitor, $S D$ standard deviation, USD US dollars

patients. As depicted in the Fig. $4 \mathrm{a}-\mathrm{c}$, the development of mean total cost due to nontreatment-related HCRU is approximately the same across the IA indications.

\section{Treatment-Related Healthcare Resource Use}

As the cost of traditional DMARDs was similar among both cyclers and persistent patients, and since it was relatively low compared to the mean total cost of biologic DMARDs $(<\$ 20$ per patient and month), traditional and biologic DMARD costs are presented together as the total cost of DMARDs. Figure 5a depicts the development of mean total cost of DMARD treatment during the 24-month measurement period, stratified by cyclers and persistent patients, respectively. Treatment costs are near identical between cyclers and persistent patients from 12 months prior to index date up to 7 months prior to index date. At this time point, treatment costs among persistent patients stabilized at around $\$ 1300$ per month, while treatment costs for cyclers started to decline. When cyclers initiated their secondline treatment, a notable increase in their average monthly treatment costs could be observed. However, subsequent to the spike, costs among cyclers started to decrease and remained consistently lower compared to persistent patients throughout the follow-up period. During the 12 months before the index date, the accumulated mean total DMARD treatment cost was significantly higher in persistent patients than in cyclers before the index date $(\$ 14,067 \quad[13,830-14,312] \quad$ vs. $\$ 12,815$ $[12,555-13,080]$, respectively). Although cyclers significantly increased their accumulated mean 

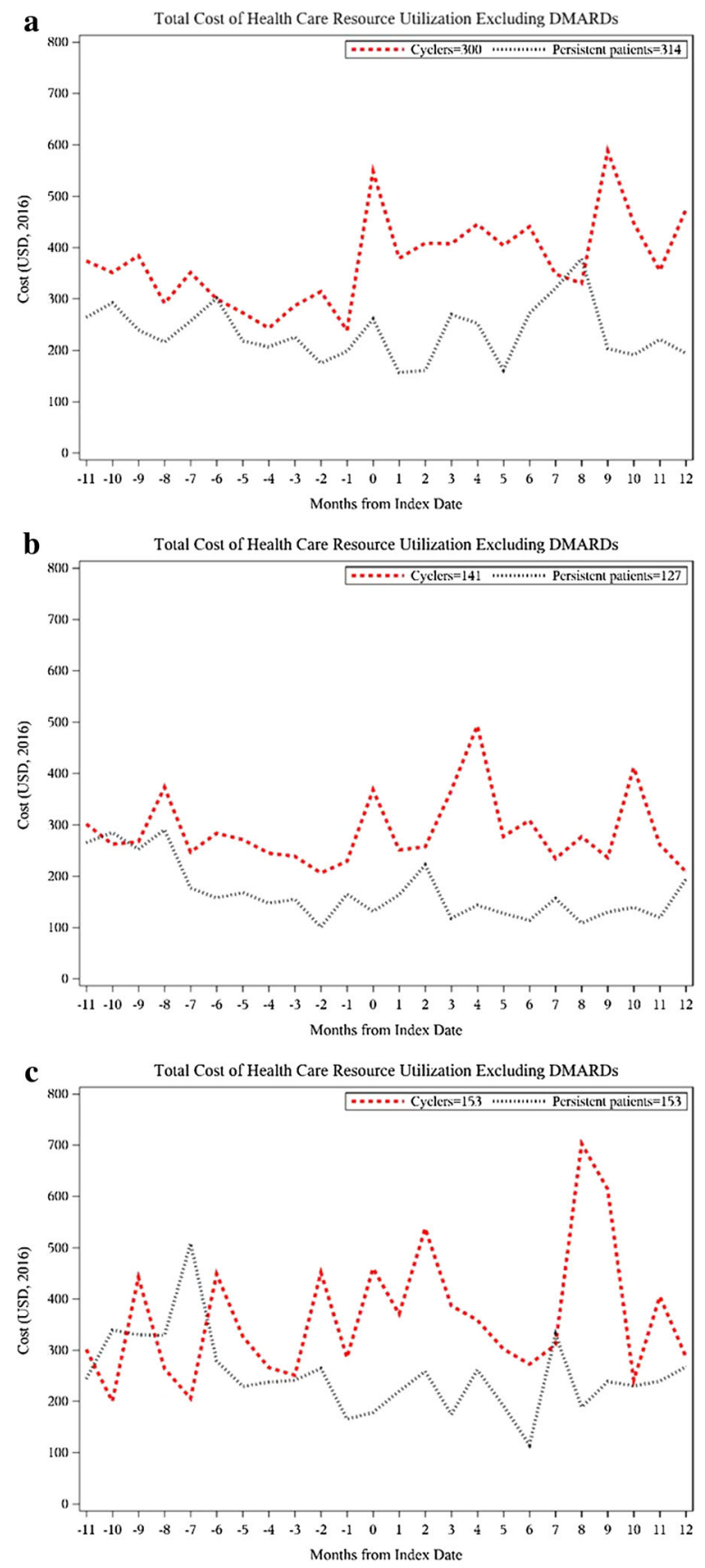

Fig. 4 Total non-treatment-related HCRU costs of propensity score-matched persistent patients and cyclers. Persistent patients and cyclers with a rheumatoid arthritis, b ankylosing spondylitis, c psoriatic arthritis

treatment costs with \$535 [70-1032] when comparing the 12 months prior to the index date to the 12 months after the index date, persistent patients continuously had higher treatment costs throughout the study time period $(\$ 15,000[14,794-15,224]$ vs. $\$ 12,815$ [12,936-13,080], respectively).

\section{Sensitivity Analysis}

To investigate the impact of second-line persistence among cyclers on treatment cost during the 12 months following the index date, a sensitivity analysis was deployed. In contrast to the main analysis, the sensitivity analysis required cyclers to remain persistent with their secondline treatment throughout the 12 months following the index date. Almost $48 \%$ of the cyclers either discontinued treatment with SCTNFi or switched to a third-line option, leaving 311 matched pairs for analysis. As illustrated in Fig. 5b, the development of monthly mean treatment costs largely concurred with the main analysis for the 12 months before index date. The difference in accumulated mean total DMARD treatment cost was also roughly the same as that seen in the main analysis $(\$ 14,156$ $[13,862-15,224]$ vs. $\$ 12,896[12,538-13,268])$. However, following the index date with the concomitant notable spike in cost, treatment costs among cyclers remained stable throughout the follow-up period, constituting a significant increase of \$3503 [3086-3934] compared to the 12 months preceding index date. Although cyclers had significantly higher accumulated treatment costs compared to persistent patients during the 12 months following the index date $(\$ 14,934 \quad[14,642-15,234]$ vs. $\$ 16,398[16,112-16,665])$, the total accumulated mean cost for DMARD treatment was similar between persistent patients and cyclers across the full 24-month follow-up period $(\$ 29,090$ vs. $\$ 29,294)$.

\section{DISCUSSION}

In this study, it was shown that among patients with IA initiating first-line SC-TNFi treatment, those who switched to second-line treatment incurred significantly higher non-treatment HCRU costs compared to those who did not experience a treatment switch during the same time period. 


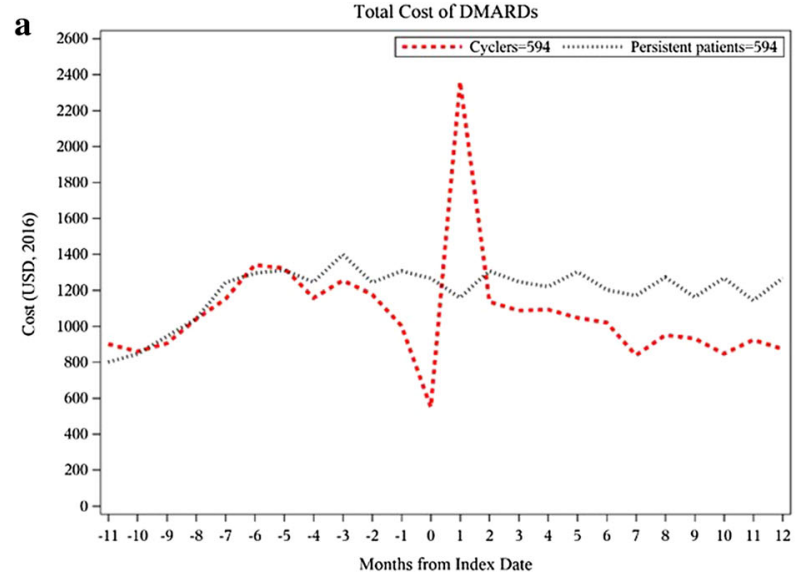

Fig. 5 Treatment-related HCRU costs of propensity score-matched persistent patients and cyclers. a Total cost of DMARDs in propensity score-matched persistent patients and cyclers. b Total cost of DMARDs resulting

Although the total costs related to nonDMARD HCRU were significantly different when comparing cyclers and persistent patients prior to index date (\$2900 vs. \$3815), costs among cyclers significantly increased while persistent patients significantly decreased their costs during the 12 months after index date (\$2587 vs. \$4637). This resulted in a statistically significant DID of $\$ 1135$ ( $\$-313$ vs. $\$ 822$ ). The difference was largely driven by costs for specialized outpatient care and inpatient care. Furthermore, it is notable that the ascending and descending cost trajectories for cyclers and persistent patients, respectively, were initiated before the index date. A possible explanation for this, which has been presented previously in a study of similar design conducted in psoriasis patients, may be that patients who are failing biologic treatment have an increase in the number of physician visits before switching treatment [26]. Consequently, some of those costs are actually attributable to the switch and, therefore, the cost impact of switching may be underestimated.

Treatment costs were higher in persistent patients before the index date, as cyclers filled less prescriptions towards the end of that 12-month period; again, it is possible that this is due to cyclers failing their first-line treatment. Despite the notable increase in treatment costs for cyclers immediately after the index date,

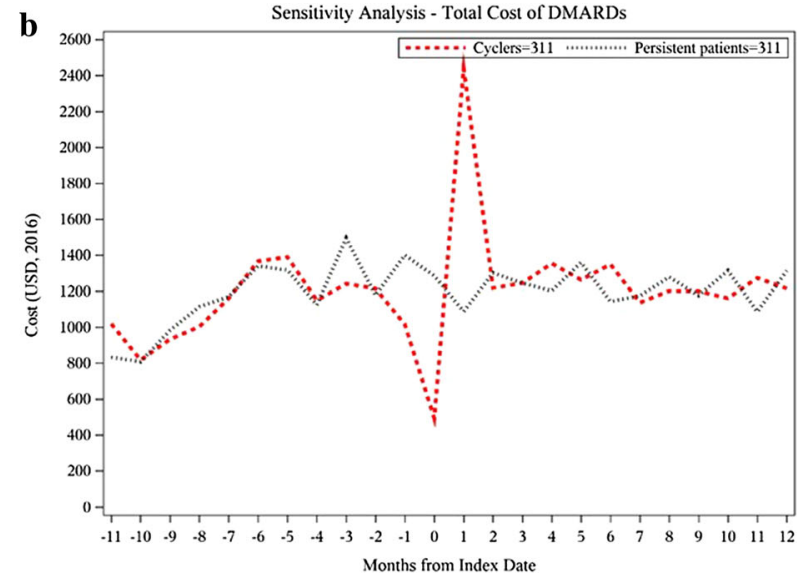

from sensitivity analysis in propensity score-matched persistent patients and cyclers

these patients had lower accumulated treatment costs during the 12 months after the index date. However, this is caused by the study design, in which all cyclers are included in the analysis regardless of their persistence with second-line treatment. During the 12 months after the index date, almost half of the included cyclers discontinued their second-line therapy and, hence, no longer incurred any treatment costs. This finding is in line with a previous study [15], in which $42-54 \%$ of patients with IA either discontinued second-line SC-TNFi treatment or switched to a third-line treatment option within 12 months of initiating second-line therapy. The plausibility of this explanation is strengthened by the fact that the sensitivity analysis, in which cyclers non-persistent with second-line treatment were excluded, showed that cyclers had higher treatment costs in the 12-month period after index date compared to persistent patients. Furthermore, while persistent patients had higher treatment costs when considering the full 24 months of follow-up in the main analysis, these were partly offset by lower costs for non-treatment-related HCRU.

Previously published studies investigating similar outcomes in patients with IA support the notion that switching SC-TNFi treatment may be associated with increased HCRU costs [27-31]. In contrast to the current study, these studies generally rely on claims data and/or 
consider patients with RA only. Our study adds to previous findings by indicating that the pattern pertaining to non-treatment HCRU costs is the same across all IA indications. In addition, the utilization of population-based administrative registers increases coverage compared to including claims data only. According to Neovius et al., the PDR register captures more than 99\% of all prescriptions of SC-TNFi in Sweden [32]. Hence, practically all IA-related use of SCTNFis in Sweden from May 6, 2010 to December 31, 2016 was considered in this study. Furthermore, the completeness and quality of the Swedish national health data registers are high $[11,12,33]$, thereby adding to the reliability of the results. An additional strength of the current study is the methodological approach used for matching patients. Switches were considered on the basis of a case-by-case data availability and follow-up, which enabled the capture and comparison of costs surrounding the date or time period of their actual occurrence. This is an update of the approach applied in our preliminary findings reported at EULAR 2018 [34], and also differs from the approaches taken in other studies presenting costs associated with switching [27-31], where stratification was based on persistence status at a given time point (e.g., 12 or 24 months) and where costs were compared over a static look back period. However, the study also has notable limitations. First, the Swedish NHDRs are administrative in nature, which means that information commonly available in disease-specific clinical registers may be difficult to derive or is lacking completely. For instance, measures of disease severity [e.g., Health Assessment Questionnaires clinical measures (HAQ) or Disease Activity Score 28 (DAS-28)] and potential reasons for switching could not be captured in the current study. Hence, these aspects of switching SCTNFi treatment could not be explored or adjusted for in any of the conducted analyses. Furthermore, the PDR does not include complete information on dosage or actual consumption of medicine. Hence, a second implication, pertaining to the dearth of clinical measures, is that estimates of persistence and switching rely on assumptions and basic information such as pack size and the derived daily dose. Consequently, dose optimization or dosing adjustments have not been accounted for. Third, the NPR does not contain any records of primary care or specialized outpatient care provided by a non-physician. For this study, the implication of this is twofold. Firstly, these healthcare resources were not captured and included in the analyses, and secondly, the comorbidities accounted for during the 12-month period prior to treatment initiation were most likely underestimated. The latter may subsequently have had an effect on the derived CCI, which consequently raises some concern around possible residual confounding since CCI was used to derive the propensity score for the PSM. Fourth, out-of-pocket payments were not captured in this study. However, those costs are likely to be limited given that the threshold for out-of-pocket payments for prescription medication in Sweden was SEK 1100 in $2016(\$ 128$, 2016). Lastly, as this study was conducted on Swedish register data, the results may not be generalizable and applicable to other settings.

Further research in this field is needed. For instance, it would be of interest to study similar outcomes using a data source containing a more comprehensive set of HCRU-associated costs, e.g., visits to primary care and indirect costs related to productivity losses due to sick leave. In addition, combining administrative data with information from clinical registers would provide a link between HCRU and disease severity, as well as potential reasons for treatment switching. This type of study would also enable persistence estimates based on consumption and more adequately capture treatment with TNFi administered intravenously. Finally, as this study was limited to SC-TNFis, similar studies including other relevant and available treatment options would be of interest.

\section{CONCLUSION}

The results show that in biologic-naïve patients treated with SC-TNFis for IA in Sweden, cyclers significantly increased their costs pertaining to non-treatment HCRU 12 months after switching treatment while persistent patients 
significantly lowered their costs during the same time period. As these findings indicate that differences in treatment persistence may have an impact on costs, further research utilizing more comprehensive data sources in alternate settings is warranted.

\section{ACKNOWLEDGEMENTS}

Funding. Sponsorship for this study, the Rapid Service and Open Access Fees were funded by Merck Sharp \& Dohme Corp., a subsidiary of Merck \& Co., Inc., Kenilworth, NJ, USA.

Authorship. All named authors meet the International Committee of Medical Journal Editors (ICMJE) criteria for authorship for this article, take responsibility for the integrity of the work as a whole, and have given their approval for this version to be published.

Prior Presentation. Preliminary findings of the results reported in this article, based on a simplified design (pertaining to matching method and the timing of cost measurements), have previously been published as an abstract and presented as a poster at the Annual European Congress of Rheumatology (EULAR) in Amsterdam, the Netherlands, 13-16 June, 2018 [34].

Disclosures. Christopher M. Black and Sumesh Kachroo are employees of Merck Sharp \& Dohme Corp., a subsidiary of Merck \& Co., Inc., Kenilworth, NJ, USA, and hold stock and options in Merck \& Co., Inc., Kenilworth, NJ, USA. Johan Dalén, Karin Luttropp, and Axel Svedbom are employees of ICON plc. ICON plc were paid consultants to Merck \& Co., Inc., Kenilworth, NJ, USA, in conjunction with the development of this manuscript. ICON plc have received funding from several pharmaceutical companies involved in the marketing products for treatment of inflammatory arthritis.

Compliance with Ethics Guidelines. As a result of the retrospective non-interventional study design and the applicable laws and regulations pertaining to the utilized data sources, informed consent and participant consent were not applicable. The study was performed in accordance with the Helsinki Declaration and, prior to initiation, ethics approval was granted by the Regional Ethical Review Board in Stockholm, Sweden (approval number 2016/1576-32).

Data Availability. The datasets generated during and/or analyzed during the current study are not publicly available due to applicable laws and regulations pertaining to Swedish administrative and clinical data available for research.

Open Access. This article is licensed under a Creative Commons Attribution-NonCommercial 4.0 International License, which permits any non-commercial use, sharing, adaptation, distribution and reproduction in any medium or format, as long as you give appropriate credit to the original author(s) and the source, provide a link to the Creative Commons licence, and indicate if changes were made. The images or other third party material in this article are included in the article's Creative Commons licence, unless indicated otherwise in a credit line to the material. If material is not included in the article's Creative Commons licence and your intended use is not permitted by statutory regulation or exceeds the permitted use, you will need to obtain permission directly from the copyright holder. To view a copy of this licence, visit http://creativecommons.org/licenses/by$\mathrm{nc} / 4.0 /$.

\section{REFERENCES}

1. Smolen JS, Aletaha D, McInnes IB. Rheumatoid arthritis. Lancet. 2016;388(10055):2023-38.

2. Ritchlin CT, Colbert RA, Gladman DD. Psoriatic arthritis. N Engl J Med. 2017;376(10):957-70.

3. Huscher D, Merkesdal S, Thiele K, Zeidler H, Schneider M, Zink A. Cost of illness in rheumatoid arthritis, ankylosing spondylitis, psoriatic arthritis and systemic lupus erythematosus in Germany. Ann Rheum Dis. 2006;65(9):1175-83. 
4. Salaffi F, Carotti M, Gasparini S, Intorcia M, Grassi $\mathrm{W}$. The health-related quality of life in rheumatoid arthritis, ankylosing spondylitis, and psoriatic arthritis: a comparison with a selected sample of healthy people. Health Qual Life Outcomes. 2009;7: 25.

5. Blum MA, Koo D, Doshi JA. Measurement and rates of persistence with and adherence to biologics for rheumatoid arthritis: a systematic review. Clin Ther. 2011;33(7):901-13.

6. Smolen JS, Burmester GR, Combe B, et al. Head-tohead comparison of certolizumab pegol versus adalimumab in rheumatoid arthritis: 2-year efficacy and safety results from the randomised EXXELERATE study. Lancet. 2016;388(10061):2763-74.

7. van der Heijde D, Ramiro S, Landewe R, et al. 2016 update of the ASAS-EULAR management recommendations for axial spondyloarthritis. Ann Rheum Dis. 2017;76(6):978-91.

8. Baser O, Ganguli A, Roy S, Xie L, Cifaldi M. Impact of switching from an initial tumor necrosis factor inhibitor on health care resource utilization and costs among patients with rheumatoid arthritis. Clin Ther. 2015;37(7):1454-65.

9. Chastek B, Becker LK, Chen CI, Mahajan P, Curtis JR. Outcomes of tumor necrosis factor inhibitor cycling versus switching to a disease-modifying anti-rheumatic drug with a new mechanism of action among patients with rheumatoid arthritis. J Med Econ. 2017;20(5):464-73.

10. Chastek B, Chen CI, Proudfoot C, Shinde S, Kuznik A, Wei W. Treatment persistence and healthcare costs among patients with rheumatoid arthritis changing biologics in the USA. Adv Ther. 2017;34(11):2422-35.

11. Ludvigsson JF, Andersson E, Ekbom A, et al. External review and validation of the Swedish national inpatient register. BMC Public Health. 2011;11:450.

12. Wettermark B, Hammar N, Fored CM, Leimanis A, Otterblad Olausson P, Bergman U, et al. The new Swedish Prescribed Drug Register-opportunities for pharmacoepidemiological research and experience from the first six months. Pharmacoepidemiol Drug Saf. 2007;16(7):726-35.

13. Askling J, Fored CM, Geborek P, et al. Swedish registers to examine drug safety and clinical issues in RA. Ann Rheum Dis. 2006;65(6):707-12.

14. Neovius M, Arkema EV, Olsson H, et al. Drug survival on TNF inhibitors in patients with rheumatoid arthritis comparison of adalimumab, etanercept and infliximab. Ann Rheum Dis. 2015;74(2): 354-60.
15. Dalen J, Svedbom A, Black CM, Kachroo S. Secondline treatment persistence and costs among patients with immune-mediated rheumatic diseases treated with subcutaneous TNF-alpha inhibitors. Rheumatol Int. 2017;37(12):2049-58.

16. Dalen J, Svedbom A, Black CM, et al. Treatment persistence among patients with immune-mediated rheumatic disease newly treated with subcutaneous TNF-alpha inhibitors and costs associated with nonpersistence. Rheumatol Int. 2016;36(7):987-95.

17. The National Board of Health and Welfare (Socialstyrelsen). Pharmaceuticals-statistics for 2014. 2015. https://www.socialstyrelsen.se/globalassets/ sharepoint-dokument/artikelkatalog/statistik/20153-17.pdf.

18. Svedbom A, Dalen J, Black CM, Kachroo S. Persistence and costs with subcutaneous TNF-alpha inhibitors in immune-mediated rheumatic disease stratified by treatment line. Patient Prefer Adherence. $2017 ; 11: 95-106$.

19. Cramer JA, Roy A, Burrell A, et al. Medication compliance and persistence: terminology and definitions. Value Health. 2008;11(1):44-7.

20. D'Agostino RB Jr. Propensity score methods for bias reduction in the comparison of a treatment to a non-randomized control group. Stat Med. 1998;17(19):2265-81.

21. Rosenbaum PR, Rubin DB. The central role of the propensity score in observational studies for causal effects. Biometrika. 1983;70(1):41-55.

22. Austin PC. Balance diagnostics for comparing the distribution of baseline covariates between treatment groups in propensity-score matched samples. Stat Med. 2009;28(25):3083-107.

23. Ho DE. Matching as nonparametric preprocessing for reducing model dependence in parametric causal inference. Political Anal. 2007;15(3):199-236.

24. Imai K, King G, Stuart EA. Misunderstandings between experimentalists and observationalists about causal inference. J R Stat Soc. 2008;171(Part 2):481-502.

25. Rosenbaum PR, Rubin DB. Constructing a control group using multivariate matched sampling methods that incorporate the propensity score. Am Stat. 1985;39(1):33-8.

26. Svedbom A, Dahlen J, Mamolo C, et al. Economic burden of psoriasis and potential cost offsets with biologic treatment: a Swedish register analysis. Acta Derm Venereol. 2016;96(5):651-7. 
27. Cannon GW, DuVall SL, Haroldsen CL, et al. Clinical outcomes and biologic costs of switching between tumor necrosis factor inhibitors in US veterans with rheumatoid arthritis. Adv Ther. 2016;33(8):1347-59.

28. Degli Esposti L, Favalli EG, Sangiorgi D, et al. Persistence, switch rates, drug consumption and costs of biological treatment of rheumatoid arthritis: an observational study in Italy. Clinicoecon Outcomes Res. 2017;9:9-17.

29. Meissner B, Trivedi D, You M, Rosenblatt L. Switching of biologic disease modifying anti-rheumatic drugs in patients with rheumatoid arthritis in a real world setting. J Med Econ. 2014;17(4):259-65.

30. Rashid N, Lin AT, Aranda G Jr, et al. Rates, factors, reasons, and economic impact associated with switching in rheumatoid arthritis patients newly initiated on biologic disease modifying anti-rheumatic drugs in an integrated healthcare system. J Med Econ. 2016;19(6):568-75.
31. Wang H, Wang Y, Michael T, Hopkins B, Bodoria M, Sondhi M, Covington M. Biologic therapy patterns and associated costs in rheumatoid arthritis patients who initiated a tumor necrosis factor antagonist over two years. Value Health. 2012;15(4):A35.

32. Neovius M, Simard JF, Askling J, group As. Nationwide prevalence of rheumatoid arthritis and penetration of disease-modifying drugs in Sweden. Ann Rheum Dis. 2011;70(4):624-9.

33. Barlow L, Westergren K, Holmberg L, Talback M. The completeness of the Swedish Cancer Register: a sample survey for year 1998. Acta Oncol. 2009;48(1):27-33.

34. Dalen J, Svedbom A, Luttropp K, Kachroo S. Costs associated with switching subcutaneous tumour necrosis factor-A inhibitor in the treatment of immune-mediated rheumatic disease. EULAR. 2018;77:527. 